- Tooth wear was a slow process in this group of patients.

- Study casts are a convenient way to monitor the longitudinal progress of tooth wear.

- Restorations are not always indicated to control tooth wear, monitoring is equally important.

- If preventing further tooth wear is more important to the patient than their appearance, monitoring is an acceptable treatment since progression is often slow.

- Treatment for tooth wear can be aggressive implementation of prevention and monitoring with study casts.

- Tooth wear in this group of patients was monitored with study casts and the progression assessed by comparing changes on the models.

\title{
Retrospective long term monitoring of tooth wear using study models
}

\author{
D. W. Bartlett
}

Objective Tooth wear is recognised as a common feature of European dentitions. However, little is known about its progression in susceptible patients. The aim of this study was to assess the degree and progression of tooth wear in patients by examining study casts taken of their teeth on two separate occasions.

Design Over 500 sets of study casts taken during an 18 -year period from patients referred for a variety of restorative procedures, were examined at Guy's Dental Hospital. Of these, 34 cases were found to have consecutive models taken at two time intervals and these were used to assess the progression of tooth wear. Study models from 19 females and 16 males, with an average age of 26 years (range 18-60) at the time of their first presentation and were all examined by a single operator. The Smith and Knight tooth wear index was used to assess the degree of tooth wear at presentation and then at another time which was a median of 26 months (interquartile range $14-50$ months) later.

Results The most common initial TWI score per surface was 1, with $54 \%$ of surfaces affected at the first assessment and 57\% at the second. Score 2 was less common (14\% at both assessments) and the scores for 3 and 4 combined were relatively uncommon with $5 \%$ of surfaces effected. Minimal progression of tooth wear was observed on study casts with only $7.3 \%$ of surfaces involved.

Conclusion In this sample, tooth wear was a slow, minimally progressive process.

Tooth wear is increasingly recognised as a common feature of European dentitions with alarmingly high levels observed in children and adults. ${ }^{1-3}$ Despite this data indicating that tooth wear is prevalent, little is known about its progression. The major problem with measuring tooth wear accurately is the identification of reproducible reference points that are themselves not eroded by acids.

An area that has shown promise on the measurement of tooth wear is laser profilometers which scan replicas of the worn

\footnotetext{
1*Dr David Bartlett, Division of Conservative Dentistry, Guy's, King's and St Thomas' Dental Institute, King's College, London.

${ }^{*}$ Correspondence to: Dr David Bartlett, Floor 25, Division of Conservative Dentistry, Guy's, King's and St Thomas' Dental Institute, Guy's Tower, London Bridge SE1 9RT

e-mail:david.bartlett @ kcl.ac.uk
}

\section{Refereed paper}

Received 13.05.02; Accepted 11.09.02

$\odot$ British Dental Journal 2003; 194: 211-213 teeth. ${ }^{4-6}$ However, their use remains within the confines of clinical research. Therefore comparing changes on study models of patients with worn teeth remains the most convenient clinical method to measure tooth wear. If the activity is recognisable from obvious changes on sequential study casts then there is justification to treat the wear with conventional restorative techniques. On the other hand if the patient's main concern is preservation of tooth tissue, reviewing the wear would be a more effective management.

Knight et $a l^{7}$ reported longitudinal measurement of wear from observations of orthodontic stone casts of patients over an average of 20 years. The authors measured occlusal/incisal wear in the pre-treatment and post orthodontic treatment records of 223 patients treated and they reported that wear could be predicted from the appearance of the study casts taken when they were in the mixed dentition stage. Pintado et $a l^{8}$ measured wear over two years on 18 adults and also reported that attrition had progressed. Yet there remains scarce information on whether tooth wear is a constantly deteriorating phenomena. The aim of this study was to assess the degree and change of tooth wear in patients over time by examining study casts taken of their teeth.

\section{MATERIALS AND METHODS}

The data for this study were derived from an extensive collection of study casts taken by dentists working at Guy's Hospital. Over 500 sets of study casts taken over an 18-year period from patients for a variety of restorative procedures, were examined at Guy's Dental Hospital by the author. Of these, there were 34 cases of tooth wear which also had consecutive models taken at two time intervals. The sample consisted of study models taken from 19 females and 16 males, with an average age of 26 years (range 18-60) at the time of their first presentation and were all examined by a single operator. A review of the notes for these subjects helped to diagnose the cause of the tooth wear.

The Smith and Knight tooth wear index ${ }^{9}$ was used to assess the degree of tooth wear at first presentation and then at another time which was a median of 26 months (interquartile range 14-50 months) later. Wear was recorded on each surface of the study cast and then the process repeated for the set taken at the different time interval. The scoring system uses five levels of severity from 0 , no wear, to 4 indicating severe wear exposing secondary dentine and the pulp (Table 1). In addition, the tooth surfaces on each 
set of models were directly compared to assess whether wear had progressed. If wear had progressed it was recorded as a positive response, no obvious wear was recorded as no change. Tooth surfaces missing or extracted between the two observations or were difficult to read were eliminated from the study.

\begin{tabular}{|c|c|c|}
\hline Score & Surface & Criterion \\
\hline \multirow[t]{2}{*}{0} & $\mathrm{~B} / \mathrm{L} / 0 / \mathrm{I}$ & No loss of enamel surface characteristics \\
\hline & C & No change in contour \\
\hline \multirow[t]{2}{*}{1} & $\mathrm{~B} / \mathrm{L} / 0 / \mathrm{I}$ & Loss of enamel characteristics \\
\hline & C & Minimal loss of contour \\
\hline \multirow[t]{3}{*}{2} & $\mathrm{~B} / \mathrm{L} / \mathrm{O}$ & Loss of enamel exposing dentine for less than $1 / 3$ of the surface \\
\hline & & Loss of enamel just exposing dentine \\
\hline & C & Defect less than $1 \mathrm{~mm}$ deep \\
\hline \multirow[t]{3}{*}{3} & $\mathrm{~B} / \mathrm{L} / \mathrm{O}$ & Loss of enamel exposing dentine for more than $1 / 3$ of the surface \\
\hline & & $\begin{array}{l}\text { Loss of enamel and substantial loss of dentine but not exposing } \\
\text { the pulp or secondary dentine }\end{array}$ \\
\hline & C & Defect 1-2 mm deep \\
\hline \multirow[t]{3}{*}{4} & $B / L / 0$ & $\begin{array}{l}\text { Complete loss of enamel, or pulp exposure, or exposure of } \\
\text { secondary dentine }\end{array}$ \\
\hline & I & Pulp exposure or exposure of secondary dentine \\
\hline & C & $\begin{array}{l}\text { Defect more than } 2 \mathrm{~mm} \text { deep, or pulp exposure or exposure of } \\
\text { secondary dentine }\end{array}$ \\
\hline
\end{tabular}

\section{STATISTICAL ANALYSIS}

The degree of tooth wear was analysed for the total number of surfaces and separately for the occlusal surfaces of the posterior teeth and the upper anterior region. The percentage of tooth surfaces with wear showed a skewed distribution and medians and interquartile ranges were calculated in addition to means and standard deviations. Wilcoxon matched-pairs signed-ranks test was used to compare the percentage of surfaces with tooth wear on study models taken on separate occasions. A separate analysis, for the percentage of surfaces exhibiting progression of tooth wear, was performed by directly comparing individual tooth surfaces on the two study models and calculating confidence intervals. Reproducibility of the tooth wear index was assessed by repeating the scoring on two separate occasions and produced a Kappa value of 0.89 .

\section{RESULTS}

The results are listed in Table 2. A total of 17,984 sites were assessed on the 68 study casts. The most common cause of tooth wear was multi-factorial but for five patients the underlying cause was not reported. The most common initial TWI score for the total number of surfaces was grade 1 with a median of 54\% of surfaces affected at the first assessment and 57\% at the second. Score 2 was less common (median of 14\% at both assessments) and the scores for 3 and 4 combined were relatively uncommon (with a median of $5 \%)$. Most of the wear observed was recorded on the anterior teeth reaching a median of 50\% at the level of grade 1, 20\% grade 2 and $8 \%$ for grade 3. Grade 1 also was the most common level to be observed on the posterior occlusal surfaces with a median of $31 \%$, 13\% for grade 2 and $0 \%$ for grades 3 and 4.

A similar pattern was observed on the second set of models with minimal changes in the intervening period. However, there was a small but statistically significant increase in the number of restored surfaces between occasions for the total number of tooth surfaces ( $p=0.023)$, and separately on the anterior surfaces at grade $1(p=0.031)$ and the posterior occlusal surfaces at grade 2 $(\mathrm{p}=0.05)$.

There was an increase in median grade 2 tooth wear between occasions on the posterior occlusal surfaces and an increase in median grade 1 tooth wear on the anterior teeth. A minor change in grade $3 / 4$ on anterior teeth approached statistical significance.

A separate analysis for the progression of tooth wear by comparing individual tooth surfaces indicated that there was a mean increase of 7.3\% of surfaces (95\% confidence interval 3.9-10.6), the anterior teeth was a mean of 5\% (2.6-7.4) and the posterior occlusal surfaces was a mean of 1.6\% (0.5-2.6).

\section{DISCUSSION}

Study casts remain the most convenient method to assess progression of tooth wear over long periods of time. The sample selected for this study represents a group of patients with known pathological tooth wear. ${ }^{10}$ It might therefore be anticipated that the tooth wear in these patients would have progressed, but this was not in fact observed. The clinicians involved in the study followed well defined criteria. If patients are not concerned about the appearance of their worn teeth and do not have intractable sensitivity, the tooth wear is reviewed before restorations are considered to establish the rate of tooth tissue loss.

Unfortunately it was not possible to determine the aetiology of the wear for each patient. However, in most cases a review of the notes showed most of the patients to have a wear multi-factorial cause of tooth wear. ${ }^{9}$ In all cases preventive advice and dietary advice was given and the advice was consistent with the advice available at the time of the review period.

One explanation for the apparent lack of progression of the tooth wear is that the condition is phasic or periodic. There is some evidence to support this from the study undertaken on reviewing tooth wear taken for orthodontic reasons suggesting suspectibility to tooth wear. ${ }^{7}$

Tooth wear indices remain the only reliable method of assessing wear over long periods of time and have proved to be effective in measuring the prevalence of wear in populations. ${ }^{1-3,11}$ The Smith and Knight index,${ }^{9}$ although used widely, does not discriminate wear particularly well once dentine is exposed. The grading of 2 and 3 (less than one third of dentine exposed and greater than one third) represents a wide variation in the severity of tooth wear but despite this it remains reasonably reproducible. This sensitivity is scarified for reproducibility. To overcome this deficiency both sets of models were compared to establish whether the wear had progressed over time. The assessment acted as a check on the index but also allowed some degree of change to be assessed between the grading levels for Smith and Knight. However, this confirmed the finding that little wear had occurred.

\begin{tabular}{|c|c|c|c|}
\hline & Baseline & Post-treatment & $P$ value for difference \\
\hline \multicolumn{4}{|c|}{ Total tooth surfaces } \\
\hline Grade 1 & 49 (20) 54 (34-67) & $49(20) 57(32-67)$ & 0.340 \\
\hline Grade 2 & $14(6) 14(11-20)$ & $15(6) 14(13-18)$ & 0.179 \\
\hline Grade $3 / 4$ & $8(11) 5(2-9)$ & $9(11) 5(2-10)$ & 0.121 \\
\hline restored & $4(6) 0(0-3)$ & $5(8) 0(0-12)$ & 0.023 \\
\hline \multicolumn{4}{|c|}{ Posterior occlusal tooth surfaces } \\
\hline Grade 1 & $31(26) 31(6-50)$ & $30(26) 25(6-50)$ & 0.275 \\
\hline Grade 2 & $15(17) 13(0-25)$ & $18(20) 13(0-31)$ & 0.050 \\
\hline Grade $3 / 4$ & $9(18) 0(0-13)$ & $11(20) 0(0-19)$ & 0.208 \\
\hline restored & $7(12) 0(0-13)$ & $8(15) 0(0-13)$ & 0.460 \\
\hline \multicolumn{4}{|c|}{ Anterior tooth surfaces } \\
\hline Grade 1 & $53(22) 50(31-70)$ & $52(21) 52(33-72)$ & 0.031 \\
\hline Grade 2 & $21(9) 20(16-30)$ & $21(10) 22(16-27)$ & 0.426 \\
\hline Grade 3/4 & $11(12) 8(2-14)$ & $12(12) 8(3-16)$ & 0.054 \\
\hline restored & $3(8) 0(0-2)$ & $5(9) 0(0-6)$ & 0.050 \\
\hline
\end{tabular}

* Wilcoxon matched-pairs, signed-ranks test 
Assessing tooth wear on study models without the benefit of direct clinical comparison is not ideal. It was, however, the only realistic way of assessing the longitudinal progression of tooth wear. Arguably, there is potential for difficulties in assessing the extent of dentine exposure without the benefit of seeing colour changes that would have been possible with a clinical examination. However, the reproducibility of the tooth wear scores was within reasonable limits and the second scoring method was aimed to overcome these concerns. Ideally, a prospective clinical comparison over a prolonged period would be helpful but may always prove to be difficult to achieve. Short term monitoring has more potential but also needs further work.

\section{CONCLUSION}

In this sample, tooth wear did not progress significantly over prolonged time periods when assessed by comparing patients' study casts. Clinically, this lends support to the concept that progression of tooth wear is not inevitable. If preventive methods are introduced or have been provided, the patients' major concerns are not the appearance of their teeth and restorations are not always indicated.
The author would like to acknowledge the statistical support and advice from $\mathrm{Dr} R$. Wilson and Mrs P Coward.

1. Bartlett D W, Coward PY, Nikkah C, Wilson R F. The prevalence of toothwear in a cluster sample of adolescent schoolchildren and its relationship with potential explanatory factors. Br Dent J 1998; 184: 125-129.

2. Smith B G N, Robb N D. The prevalence of toothwear in 1,007 dental patients. J Oral Rehab 196; 23: 232-239.

3. Al Dlaigan Y H, Shaw L, Smith A. Dental erosion in a group of British 14-year-old school children. Part 1: Prevalence and influence of differing socioeconomic backgrounds. Br Dent J 2001; 190: 145-149.

4. Chadwick R G, Mitchell H L, Cameron I, Hunter B, Tulley M. Development of a novel system for assessing tooth and restorations wear. J Dent 1997: 25: 41-47.

5. Bartlett D W, Blunt L, Smith B G N. Measurement of tooth wear in patients with palatal erosion. Br Dent J 1997; 182: 179-184

6. Azzopardi A, Bartlett D W, Watson T F, Sherriff M. The protection given by dentinebonding agents to prevent erosion - an in vitro study. Caries Res 2000; 34: 5.

7. Knight D J, Leroux B G, Zhu C, Almond J, Ramsey D S. A longitudinal study of tooth wear in orthodontically treated patients. Am J Orthod Dentofac Orthop 1997; 112: 194-202.

8. Pintado M R, Anderson G C, DeLong R, Douglas W H. Variations in tooth wear in young adults over a two-year period. J Prosthet Dent 1997; 77: 313-320.

9. Smith B G N, Knight J K. An index for measuring the wear and tear of teeth. Br Dent J 1984; 156: 435-438.

10. Smith B G N, Knight J K. A Comparison of patterns of tooth wear with aetiological factors. Br Dent J 1984; 157: 16-19.

11. Lussi A. Dental erosion; clinical diagnosis and case history taking. Eur J Oral Sci 2002; 104: 191-198. 